\title{
Errata
}

\section{Adsorption and Degradation of Benfuracarb in Three Soils in Hunan, People's Republic of China}

N. Xue, ${ }^{1,2}$ R. Yang, ${ }^{1}$ X. Xu, ${ }^{2}$ H. M. Seip, ${ }^{3}$ Q. Zeng ${ }^{1}$

${ }^{1}$ Department of Environmental Sciences and Engineering, Hunan Agricultural University, Changsha 410128, People's Republic of China

2 State Key Laboratory of Environmental Chemistry and Ecotoxicology, Research

Center for Eco-Environmental Sciences, Chinese Academy of Sciences,

18 Shuangqing Road, Beijing 100085, People's Republic of China

${ }^{3}$ Department of Chemistry, University of Oslo, Post Office Box 1033, Blindern, 0315

Oslo, Norway

Q. Zeng's name was inadvertently misspelled in this article [Bull Environ Contam Toxicol (2006) 76:720-727, DOI: 10.1007/s00128-006-0979-x]. The corrected list of authors' names is printed above. 